\title{
Galaxy build-up at cosmic dawn: Insights from deep observations with Hubble, Spitzer, and ALMA
}

\section{Pascal Oesch}

\author{
University of Geneva, Switzerland
}

\begin{abstract}
Over the last few years, great progress has been made in understanding the buildup of the first generations of galaxies based on deep optical and near-infrared imaging from the Hubble Space Telescope. However, HST only samples the rest-frame UV light of galaxies at $z \geqslant 4$, providing only limited information on the dust obscuration and on stellar masses of these sources. Fortunately, several Spitzer/IRAC programs have complemented the extragalactic HST fields with ultra-deep imaging data, allowing for a rest-frame optical view on early galaxies. Together with first ALMA/ NOEMA (sub)mm observations on distant galaxies, we are starting to gain a more and more complete picture of galaxy star-formation and mass build-up in the early universe. In this talk, I will present an overview of our current understanding of normal star-forming galaxies at $z>3$ based the combination of HST+Spitzer+ALMA/NOEMA data. In particular, I will show how HST as already pushed into JWST territory with the discovery and spectroscopic confirmation of a galaxy at $z=11.1 \pm 0.1$, only $\sim 400$ Myr after the Big Bang. I will also highlight some of the exciting possibilities that lie ahead with JWST to push the spectroscopic frontier to the cosmic dawn and to finally probe the physics of early galaxies.
\end{abstract}

